\title{
Re-engaging Students by Teaching from the Middle and Back of a 500- seat Lecture Hall
}

\author{
James Andrew Smith \\ Lassonde School of Engineering, York University, Toronto, Canada \\ drsmith@yorku.ca
}

\begin{abstract}
To better engage with students, especially in a large traditional classroom, one should consider teaching from multiple locations within the classroom, not just in the front. In classes where computers can be used to project material to the front of the classroom teachers should consider wireless peripherals or computers to achieve dynamic content delivery from beyond the podium. A variety of technologies were examined here, with wireless Wacom drawing tablets paired with either Apple or Windows computers, Miracast-enabled Windows tablets, and Doceri's Whiteboard mode on Windows tablets and Apple iPads yielding the best results. The Wacom Tablet and Apple Trackpad were found to enable greater engagement of students, from the front to the rear of the classroom. More rigorous tests with the other technologies is to be carried out in the future.
\end{abstract}

Keywords: screen-casting, mobile teaching, tablet, active teaching, Doceri, TeamViewer, trackpad, Wacom tablet, interactive whiteboard, PowerPoint.

\section{INTRODUCTION}

In a 500-seat lecture hall, how can a single professor capture the attention and imagination of students with the constant droning of dozens of whispered conversations and the flickering glow of hundreds of phones, tablets and laptops? [1, 2, 3] In the Fall 2016 offering of an introductory programming class at York University these issues were derailing the class. This introductory programming class, offered to all first year engineering students at York University, used to be offered in two lecture sections of 200-300 students. For the 2016-2017 academic year the course was offered in a single section, with classes held in a 500-seat auditorium. While attendance was generally good (approx. 75\%), it was clear that students were having trouble concentrating in class. Feedback from faculty observer colleagues highlighted how the noise level in the classroom was making it especially difficult for students in the rear half of the class to engage in meaningful learning. In the words of my colleague, I had "given up on teaching" to the back half of the class. I hypothesized that if I could get physically closer to these students I might be able to reengage them. But since teaching material was tied to the computer and podium's projection equipment at the front of the classroom, how could the teaching flow be maintained if I was physically separated from the podium and not so distant (literally!) from my students? The answer lay in selecting an appropriate wireless technology. In my case, I chose to alternate between using an Apple Magic Trackpad 2 and a Wacom Intuos tablet to permit dynamic changes to the presentation material while moving throughout the classroom. While student engagement went up as a result of the teaching no longer being constrained to the front of the classroom, other wireless tools could also have been used. Here, I look at alternatives that could be used in large classrooms by both myself and others, at York University and elsewhere.

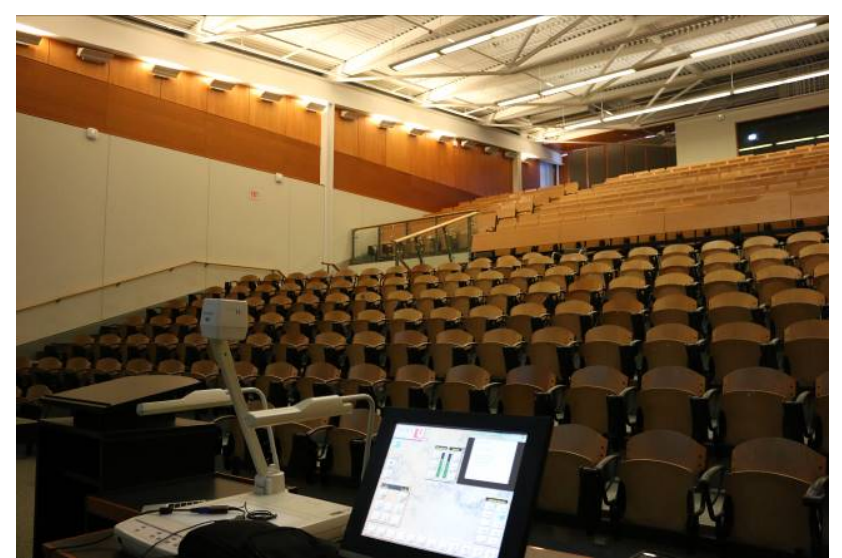

Figure 1 A 500-seat lecture hall at York University.

For presenting information to my students I refrain from using the whiteboard/blackboard, as turning my back to my students while talking with them runs counter to my goal of increasing engagement. Furthermore, using a computer and projector provides flexibility with augmented, dynamic notes and audio-visual resources. Like many university and college professors I occasionally use Microsoft's PowerPoint but prefer to use tools like Mage Software's InkBook (a Macintosh-only free-form note-taking software similar to Microsoft's 
OneNote or the Whiteboard mode in SP Control's Doceri) that encourage a more dynamic form of delivery. I tend to use a Wacom Intuos tablet to draw on the InkBook or PowerPoint slides, effectively augmenting my slides with traditional free-form blackboard functionality. I can use this tablet from about $10 \mathrm{~m}$ away from the podium. I also use a wireless Apple Magic Trackpad 2 to navigate through material when roaming the classroom and interacting with students.

The objective of this paper is to explore practical solutions for permitting the teacher to present interactive electronic material from anywhere in a large theatre-style classroom -- not just the podium at the front. The motivation for untethering the teacher from the front-ofclassroom podium is the observation that students pay more attention when the teacher is not restricted to simply lecturing from a single location. Here, I will explore alternative technologies beyond the Wacom Intuos drawing tablet and Apple Magic Trackpad 2 for both Windows and Apple computers.

\section{METHODOLOGY}

This paper focuses on the testing of wireless technologies for teaching in a large classroom. The front of the room is equipped with a large blackboard and a podium connected to a pair of projectors. The podium has a Dell Windows desktop PC embedded and is also equipped with a VGA port for connection to mobile devices such as laptops. This setup is typical of many large classrooms in colleges and universities in North America. Two mobile computers are also used in these tests, an Apple MacBook Air and an HP Elite x2 1012. One test is conducted with a $4^{\text {th }}$ generation iPad.

The rear of the classroom, shown in Fig. 1, is about five meters higher than the front of the classroom and the distance from the podium to the rear of the classroom is about 24 meters. A walkway is found about one-third of the way from the front to the rear of the room. There is seating for approximately 500 students.

As is typical for many classrooms, it is expected that all visual content is to be delivered via projections at the front of the classroom. While the projection of static content (typical of PowerPoint-style slides) is acceptable, a more dynamic approach to content delivery, including free-form writing or, at least, live annotations of PowerPoint slides.

Two categories of technologies were tested in an empty lecture theater: internet-mediated wireless (e.g. screen- sharing applications) and non-internet-mediate wireless (e.g. presentation remotes).

In most scenarios, an Apple MacBook Air laptop computer was directly connected to the classroom projection system. A variety of wireless devices were used to manipulate this computer and the projected images from it. In most tested scenarios the Apple laptop could have been replaced with a Windows laptop or desktop such as those often built directly into the classroom podiums.

Internet-mediated wireless technologies include applications like Skype that require your computer to use the internet before it can communicate with another device. Here, we evaluated two software products that permit the screen of one computer to be shared with another computer: TeamViewer and Doceri. [4, 5] In both cases the tests were only conducted in an empty classroom.

TeamViewer is commonly used in technical support applications whereby a technician can both view and control a remote client's computer over the internet. A number of operating systems for both local and remote machines are supported, including Windows, Mac OS, Linux and iOS. The test configuration involved a "local" Windows 10 tablet (the Microsoft Surface-like HP Elite $\mathrm{x} 2$ 1012) controlling "remote" MacBook Air laptop connected to the classroom's podium and projectors. The connection between the two computers was established via TeamViewer's servers over York University's Wi-Fi network. The connection process took less than 30 seconds.

The Doceri test was conducted twice. In the first case the controlling computer was a Windows 10 tablet (an HP Elite $x 2$ 1012), while in the second case the controller computer was an Apple iPad (4th generation). In both cases the remote computer was an Apple MacBook Air laptop connected to the classroom's podium and projectors. The connection between the two devices was established via SP Control's servers over the York University's Wi-Fi network. Doceri requires that the two computers be on the same network when connecting, as is typical in a university or college. The connection process took under 30 seconds. Both full screen sharing and whiteboard modes were tested. During screen-sharing the MacBook Air was running PowerPoint and projecting onto a pair of projectors to the front of the classroom. Navigation and annotations were possible using both the mobile iPad and mobile Windows 10 tablets. Lag was noticeable when attempting to annotate the PowerPoint slides. 
The whiteboard mode in Doceri was also tested using the MacBook Air as the display computer and the iPad and Windows 10 tablets as mobile controlling computers. When annotating / drawing on the mobile computers, no noticeable lag was noticed on the iPad or Windows 10 machines, nor on the image displayed by the remote Apple MacBook Air.

Other tools were tested using non-internet-mediated technologies. None of these technologies require a live connection to the internet in order to function. These tools include a relatively standard presentation remote (SMK-Link Global Presenter), a trackpad (Apple Magic Trackpad 2), a drawing tablet (Wacom PTH-651), and a tablet computer (HP Elite X2 1012) with Miracast technology. Full-size wireless keyboards with integrated trackpads or trackballs, like the IOGear GKM561R were not examined, as they cannot be used without a table.

The SMK-Link Global Presenter (VP4350) is a $2.4 \mathrm{GHz}$ standard presentation remote with a $30 \mathrm{~m}$ advertised range. It was tested from a variety of positions in the classroom to navigate and annotate PowerPoint slides on both an Apple (MacBook Air) and Windows 10 (HP Elite x2 1012) computers connected to the classroom projection system. The range of the presenter was only tested in an empty classroom.

Many mobile computers come with wireless display technology built-in. Miracast is the most common standard on Windows 10 machines, while Airplay is the name given to the equivalent Apple technology. For this exercise, the Miracast system was tested using a Window 10 tablet and a Belkin Miracast adapter (F7D750v1) connected to the VGA port of the classroom's projector system. No special software needed to be added as it was already embedded the Belkin adapter and the Windows 10 machine (HP Elite X2 1012). No additional computer was required. Setup took about a minute, consisting primarily of setting the tablet to seek the specific Belkin adapter and to complete the handshaking routine. While the Belkin adapter supports HDMI output the specific classroom used only supported VGA projectors. An inexpensive HDMI to VGA adapter was required to permit this. This setup was only tested in an empty classroom.

A Wacom Intuos tablet [6] was next used to gauge its ability to send content from anywhere in the lecture hall to a computer at the podium. The wireless capability of the Wacom Intuos Pro (Medium; PTH-651, with optional INF-A068 and INF-A069 wireless transceivers) was tested on both the Apple MacBook Air and HP Elite X2 1012 directly wired to the classroom projector. The range of the tablet was tested in both an empty classroom and a full classroom.

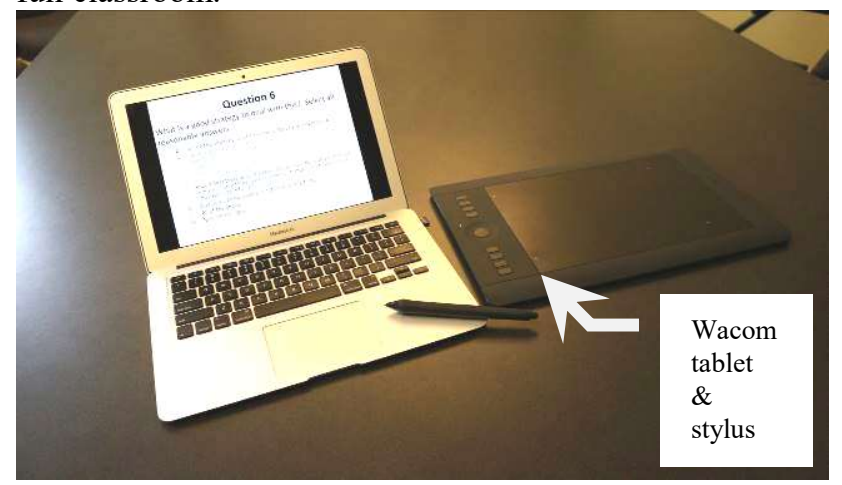

Figure 2 Wireless Wacom Intuos Pro tablet (right) for annotating PowerPoint slides on a Macintosh laptop (left).

The Apple Magic Trackpad 2 was tested on the Apple MacBook Air directly wired to the classroom projector. A software application, TenOne Design's Inklet 2, was added to permit the trackpad to be used for annotations and navigation in PowerPoint and Mage Software's InkBook. The range of the trackpad was tested in both an empty classroom and a full classroom.

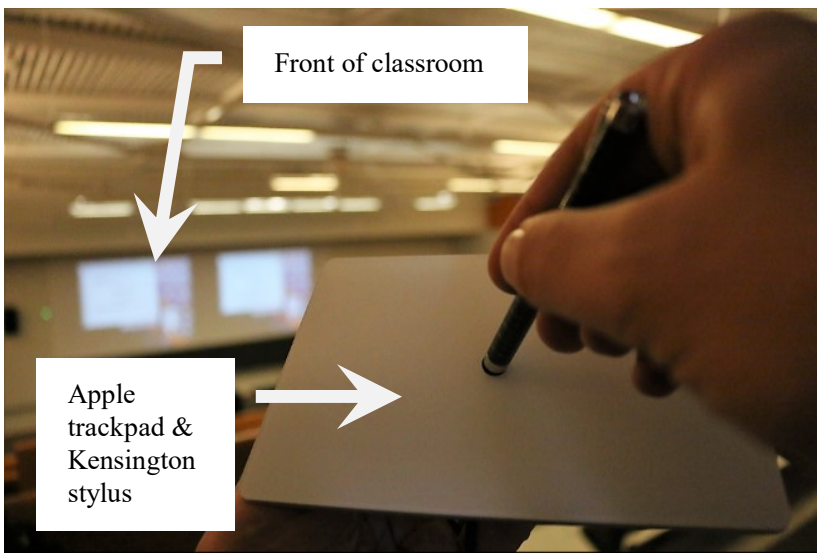

Figure 3 Apple trackpad combined with a stylus and Inklet 2 software (foreground) to annotate PowerPoint content (background) from the rear of a classroom.

\section{RESULTS}

During the tests of internet-mediated wireless approaches, the whiteboard-mode Doceri tests were considerably more responsive. Doceri's whiteboard mode was also tested using the MacBook Air as the display computer and the iPad and Windows 10 tablets as handheld mobile computers from a variety of locations in the classroom. When annotating / drawing on the mobile computers, no noticeable lag was noticed on the iPad or Windows 10 machines, nor on the image displayed by the remote Apple MacBook Air. 
In contrast, the TeamViewer tests were found to be less responsive, with noticeable lag. This is because the display computer (the Apple MacBook Air connected to the classroom's projectors) permits so much data to be transferred to the mobile computer (Windows 10 tablet), requiring a lot of network bandwidth. While the lag (up to a few seconds) did not negatively affect the navigation through PowerPoint slides, it made live annotation of those slides very difficult. Lines and curves appeared inconsistently because of this.

The first non-internet-mediated device tested, the SMK Global Presenter, was found to be reliable throughout an empty classroom, at distances of up to $24 \mathrm{~m}$ (the $30 \mathrm{~m}$ maximum range was greater than the size of the classroom). This remote is similar to countless PowerPoint remotes and it performed with little noticeable lag throughout the classroom. The built-in laser pointer is a useful addition. The integrated joystick, similar to the pointing stick integrated in Lenovo/IBM ThinkPad laptops, worked well for one-handed navigating PowerPoint slide decks, but is too slow and awkward for extensive slide annotations.

With the Belkin Miracast adapter, PowerPoint was tested using a Windows 10 tablet, both in terms of navigation and it terms of annotation using the tablet's stylus. No lag was noticed between application of the stylus on the tablet and the projected result. Distances of up to $24 \mathrm{~m}$ were tested, in an empty classroom. It is assumed that in a full classroom the range will be shorter as Miracast technology uses the same frequencies as WiFi networking and so may suffer from interference from student Wi-Fi enabled devices.

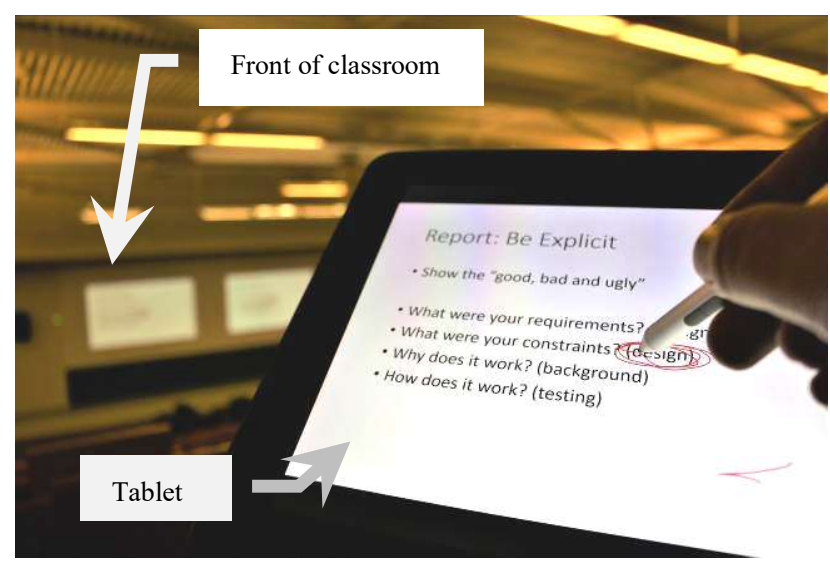

Figure 4 Windows 10 tablet (foreground) annotating PowerPoint slides from the rear of a 500-seat lecture hall using Miracast wireless technology.

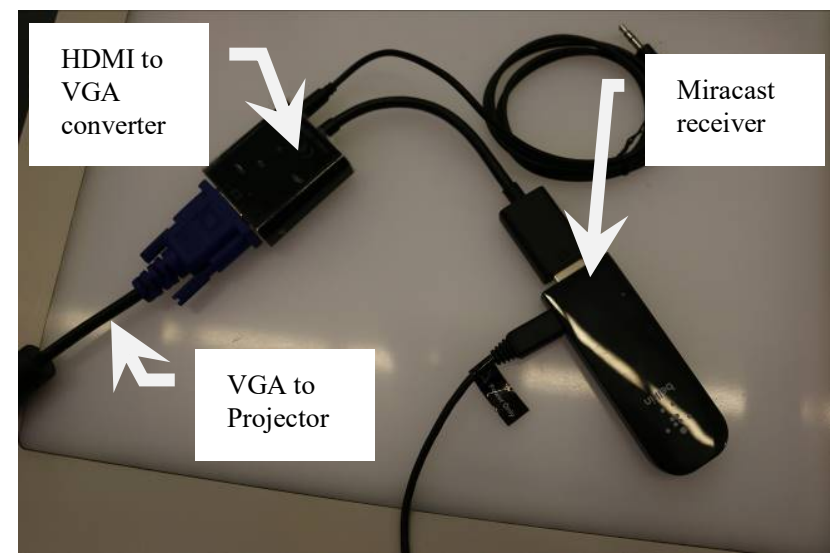

Figure 5 Belkin Miracast adapter connected to the projector's VGA input. Setup was quick and lag was minimal when used with a Miracast-equipped Windows 10 tablet.

A Wacom Intuos Pro Medium drawing tablet was tested wirelessly with both a Windows 10 tablet and an Apple laptop. It was capable of navigating PowerPoint slides on both machines. Furthermore, it was capable of annotating slides with its included stylus. On the MacBook Air it could be used for free-from writing and annotation existing slides in InkBook, a notebook-like application that is similar to the whiteboard mode in Doceri. A range of up to 24 meters in an empty classroom was tested. This was the first technology tested and only one of two tested when the classroom was full. Reliable operation was found to be up to about $10 \mathrm{~m}$ in a full classroom. Response of the students to having me teach with the drawing tablet from middle and near the back of the classroom was very positive, with students noticeably more engaged.

In a similar fashion to the Wacom Intuos, but limited to use with the Apple MacBook Air, an Apple Magic Trackpad 2 combined with the Ten1 Design's Inklet 2 application and a Kensington stylus. Navigating PowerPoint or Mage Software's InkBook slides was possible using the virtual sliders mapped to applicationspecific keys for moving forward and backward through the slide deck. The pressure sensitivity of the trackpad made it possible for the Inklet 2 application to distinguish between cursor navigation and annotation tasks. Using it to annotate is less intuitive than with the Wacom and it was easier to annotate with a finger in Inklet than it was with a stylus. A range of up to 24 meters in an empty classroom was tested. This was the second technology to be tested within a full classroom. Reliable operation of the trackpad was found to be up to about $10 \mathrm{~m}$ when the classroom was full, but was still mostly usable near the rear of the classroom. As with the Wacom tablet, when I 
used this to teach from the middle and rear of the classroom students were noticeably more engaged.

\section{DISCUSSION}

One-handed presentation remotes are popular because of their simplicity and reliability. The long-range SMK Global Presenter ( $\mathrm{p} / \mathrm{n}$ VP4350) shares this characteristic with other presentation remotes. While it is good at simply navigating through PowerPoint slides on Windows 10 or Macintosh computers it is not very capable of permitting annotation capability via its joystick.

The Apple Magic Trackpad 2 (p/n A1535) performed well for navigating through slides in PowerPoint and Mage Software's InkBook on a Macintosh laptop. The use of Ten1 Design's Inklet 2 software permitted drawing-tablet functionality similar to Wacom tablets. The navigation and annotation functions were more awkward to use than the Wacom tablet, described below. After a discussion with the Inklet 2 developers an update to the software (2.0.2) was released but there was insufficient time to test the update prior to the deadline for this manuscript.

The lightweight nature of the Wacom tablet, combined with the ease of setup, its ability to reliably distinguish between fingers and stylus, the almost paper-like sensation between stylus and surface and configurable buttons for navigation, etc. make it a superior choice for use with applications such as PowerPoint, OneNote, InkBook and others. A new version (2017) of the Intuos tablet replaces the proprietary $2.4 \mathrm{GHz}$ wireless USB dongle in the PTH651 model with a Bluetooth 4.2 system that, while untested, will likely be more practical on computers without USB-2 ports. The minor drawback with this approach, as opposed to the Miracast or Doceri approach, is that the lack of direct visual feedback on the drawing tablet leads to an initial discomfort ([7], https://www.youtube.com/watch?v=BOOgPUEiBDo). A short period of practice (a few days to a few weeks) will aid in overcoming this dissonance. The Wacom tablet and Apple trackpads were the only ones tested in a full classroom and students were noticeably more engaged when I used them while moving throughout the classroom.

While heavier to use than a Wacom drawing tablet, a Miracast-enabled Windows 10 tablet (the HP Elite x2 1012 -- similar to the Surface Pro 4) was found to be very reliable for navigating PowerPoint slides, as well as annotating them. Lag was nonexistent on the tablet itself and minimal on the projected image. The Belkin Miracast receiver was straight-forward to use and was found to be reliable when tested in an empty classroom.
Relying on a university or college's networking hardware to establish a connection between the remote computer and the controlling computer is risky. It is unlikely that the network, or the edge nodes around a classroom have been stress tested for hundreds of students. Therefore, internet-enabled solutions like TeamViewer and Doceri (that were tested), or untested alternatives such as Skype screen sharing, RealtimeBoard, Sketchboard.io, Rowmote, Conference Pad, and Astropad are risky in that they might not be reliable. Furthermore, latency issues can make such solutions unusable in anything other than simple slide projection applications. TeamViewer's and Doceri screen sharing modes both suffered from latencies of up to five seconds. These kinds of latencies make it very difficult to annotate slides or write freehand with a stylus in a classroom setting.

Doceri's whiteboard mode, on the other hand, overcame the latency issue. The whiteboard on the iPad was mirrored on the remote Mac laptop connected to the overhead projector. While there was occasionally up to a few seconds of latency on the remotely-projected image, the local latency on the iPad was barely noticeable. The Doceri software provides further options for recording presentation material, whiteboard mode provides a wide variety of graph-paper templates and slides can be exported.

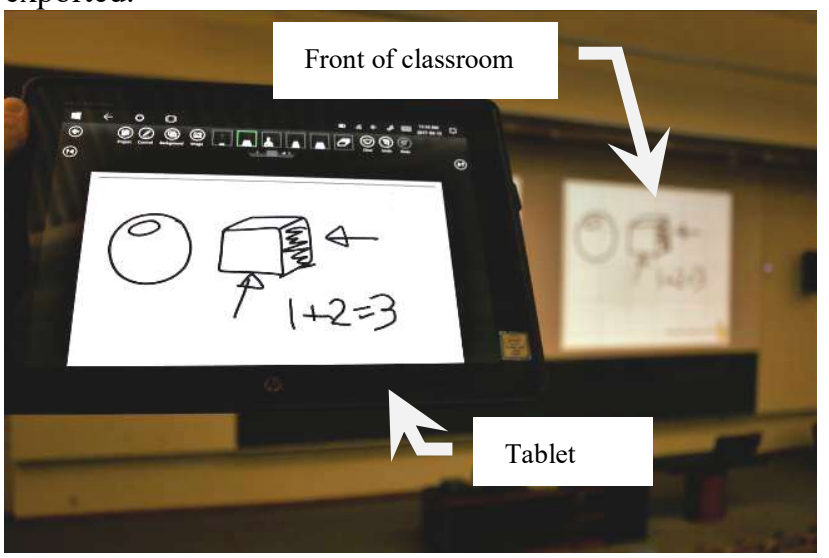

Figure 6 Doceri whiteboard mode on a Windows tablet (left, foreground) and classroom projected image (right, background).

The software and hardware solutions examined here do not consist of an exhaustive list but, rather, a sampling of representative technologies. A more in-depth study could be carried out with more technological options and in occupied classrooms.

\section{CONCLUSIONS}

The objective of this study was to test technologies which could enable the teacher to present material via a projector 
from anywhere in a large (500 seat) lecture theater. The rationale is that, based on personal experience, being able to roam the classroom enables better engagement of students. Depending on the state of the classroom's internet infrastructure, teacher's style of delivery and tolerance for software and hardware setup, there are different optimal technologies that can help with this. All solutions presented here were tested and were shown to be capable of being used up to $24 \mathrm{~m}$ from the front of an empty 500 seat lecture theater. Only the Apple trackpad and Wacom tablet were tested in a full classroom, and operation of both was found to permit reliable use to about the middle of the classroom (about 10m) and student engagement was noticeably improved with both options.

There is no single right way or wrong way to approach mobile teaching in a large classroom. However, there are key components that a good solution must address: the mobile device needs to be lightweight since it could be held in your hands for a lengthy period of time. Lag between mobile input and projected image shouldn't be noticeable, or at least under a second to not interfere with the teaching flow. Setup time should be no more than a few minutes and should not require any complex details. Ideally, the solution should not be dependent on external resources such as off-campus servers or a wireless infrastructure that could be unreliable due to radio saturation and other factors.

A long-range variant of countless presentation remotes, the one-handed SMK Global Presenter (VP4350) is the simplest tool for simply navigating through PowerPoint slides on Windows 10 or Macintosh computers with only rudimentary annotation capability via its joystick.

Simple wireless trackpads were also examined. Both the Apple Magic Trackpad 2 (p/n A1535) and the Wacom Intuos Pro Medium (p/n PTH-651) were tested and the Wacom tablet was found to be superior for both navigating and annotating slides in applications such as PowerPoint, OneNote and Mage Software's InkBook. Further tests using the newly-released Inklet v2.0.2, as well as the Bluetooth-enabled Wacom Intuos trackpads are planned.

If one finds the Wacom tablet to be insufficiently capable, then a heavier Windows 10 or iPad screen-enabled tablet could be considered. Using Miracast is an elegant solution that only requires a simple adapter (Belkin F7D750v1) and a Miracast-enabled Windows 10 tablet to permit showing students everything on your tablet, all without relying on the university's network infrastructure. Alternatively, Apple users can use equivalent Airplay technology, while Chromecast is available for Android devices. When the university's networking infrastructure can be relied upon, and a second, fixed computer is available at the podium to project images, then the whiteboard mode in Doceri should be considered as it was found to work responsively with both Windows 10 and iPad tablets.

\section{Acknowledgements}

Funding for the purchase of the equipment used in these tests is from York University's Lassonde School of Engineering Dean's Office. This project was initiated based on a conversation with Dr. Maria Wallis, a fellow participant in York University's Teaching Commons' "Teaching Wheels" program, organized by Dr. Barbara Kerr. Many thanks to Dr. Kerry Doyle for her observations regarding the IOGear GKM561R wireless keyboard.

\section{References}

[1] Elisa Carbone. "Students behaving badly in large classes." New Directions for Teaching and Learning 1999, 77, pp. 3543, 1999.

[2] Faria Sana, Tina Weston, and Nicholas J. Cepeda. "Laptop multitasking hinders classroom learning for both users and nearby peers." Computers \& Education vol 62, pp 24-31, 2013.

[3] Andrew Robinson "Even More Laptops In The Classroom - Once More Unto The Breach, Dear Friends." , URL: https://medium.com/precarious-physicist/even-morelaptops-in-the-classroom-c511b6ba75b5, April 2017. (Last viewed April, 2017)

[4] Stepan Hubalovsky. "Remote Contact Learning as Method of Teaching of Algorithm Development and Programming in Distance Study." Procedia-Social and Behavioral Sciences vol 191, pp. 1958-1963, 2015.

[5] Lee J. Silverberg, John Tierney, and Matthew J. Bodek. "Use of Doceri software for iPad in online delivery of chemistry content." Journal of Chemical Education, vol 91, no. 11, pp. 1999-2001, 2014.

[6] Marisa Llorens, Edmund Nevin, and Eileen Mageean. "Online resource platform for mathematics education." Frontiers in Education Conference (FIE), 2014.

[7] Paul Zdepski, "Going Digital"; Thesis Project, Hartford Art School, University of Hartford; URL: http://www.zillustration.com/ downloads/Zdepski Thesis F inal_Web.pdf (Last viewed April, 2017) 